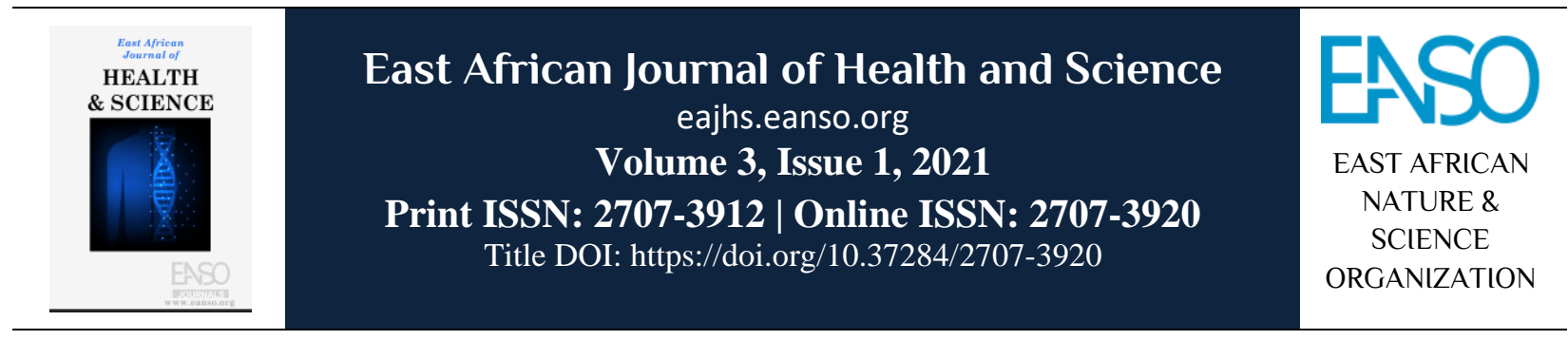

Original Article

\title{
Addressing the Healthcare Needs of African Men who have Sex with Men: Barriers to Healthcare and Promoting HIV and STI Treatment in Sub- Saharan Africa.
}

\author{
Michael W. Ross ${ }^{1 *}$, John Kashiha ${ }^{2}$,Lourence Misedah ${ }^{3}$, Lucy R. Mgopa ${ }^{4}$, Markus Larsson ${ }^{5}$, \\ Anette Agardh ${ }^{5}$, \& Krishna Kumar Venkitachalam ${ }^{6}$ \\ ${ }^{1}$ Program in Human Sexuality, University of Minnesota Medical School, MN 55454, Minneapolis, USA. \\ ${ }^{2}$ CHESA, Dar es Salaam, Tanzania, P. O. Box 114, Malik Street, Tanzania. \\ ${ }^{3}$ School of Public Health, University of Texas, 1200 Pressler Street Houston TX 77030, USA. \\ ${ }^{4}$ Department of Psychiatry, Muhimbili University of Health and Allied Sciences, P.O. Box 65001, Dar es Salaam, Tanzania. \\ ${ }^{5}$ Division of Social Medicine and Global Health, Department of Clinical Sciences, Lund University, P.O. Box 187, Lund, SE 221 \\ 00, Sweden Malmö, Sweden. \\ ${ }^{6}$ Freelance Medical Research Consultant and Writer, Mumbai, India (affiliated to Board of Editors in Life Sciences) \\ *Author for correspondence ORCID ID: https://orcid.org/0000-0002-5718-9989; email: mwross@umn.edu.
}

Article DOI: https://doi.org/10.37284/eajhs.3.1.339

\section{Date Published: ABSTRACT}

08 June 2021 Barriers to healthcare for men who have sex with men (MSM) in sub-Saharan Africa (SSA) are rooted in stigma and discrimination against MSM fuelled by the

Keywords: criminalization of homosexuality. These barriers are influenced by factors

Healthcare Needs, involving MSM and the healthcare workers (HCWs). MSM are uncomfortable

Healthcare disclosing their identities and conceal symptoms of sexually transmitted infections

Worker,

Men Who Have (STIs), especially those in areas associated with homosexual behaviour to avoid stigmatization. MSM experience ill-treatment and abuse by HCWs and have Sex With Men,

HIV And STI

Treatment,

Sub-Saharan concerns regarding HCWs maintaining confidentiality and privacy. This forces MSM to seek care from pharmacies/drugstores or resort to self-treatment, which may not be effective. This, coupled with increased HIV- and STI-risk behaviours resulting from depression and internalized homophobia, result in further spread of

Africa STIs. Further, barriers in HIV testing lead to exclusion of MSM from treatment cascades. Insufficient sexuality training of HCWs leads to discrimination or denial of treatment for MSM, particularly in government-run clinics. The criminalization of homosexuality deters HCWs from offering treatment to MSM. The prevailing discriminatory environment that accompanies the criminalization of homosexuality forces HCWs to don the role of moral and legal enforcers, making them the perpetrators of forced anal examinations that continue to be prevalent in SSA. Nevertheless, there is evidence to show HCWs in SSA recognize their lack of

59 | This work is licensed under a Creative Commons Attribution 4.0 International License. 
training in sexuality and have welcomed educational opportunities to better understand the healthcare needs of MSM. Pilot educational interventions for HCWs in SSA have been received enthusiastically, and have resulted in significant changes in HCW knowledge and attitudes and dealing with MSMs with STIs. This work reviews existing literature on barriers to STI-related healthcare for MSM in SSA. By drawing parallels to barriers that were overcome in the HIV epidemic in the 1980s, suitable solutions focusing on HCW education are suggested.

\begin{abstract}
APA CITATION
Ross, M. W., Kashiha, J., Misedah, L., Mgopa, L. R., Larsson, M., Agardh, A., \& Venkitachalam, K. K. (2021). Addressing the Healthcare Needs of African Men who have Sex with Men: Barriers to Healthcare and Promoting HIV and STI Treatment in Sub-Saharan Africa. East African Journal of Health and Science, 3(1), 59-77. https://doi.org/10.37284/eajhs.3.1.339.
\end{abstract}

\title{
CHICAGO CITATION
}

Ross, Michael W., John Kashiha, Lourence Misedah, Lucy R. Mgopa, Markus Larsson, Anette Agardh and Krishna Kumar Venkitachalam. 2021. "Addressing the Healthcare Needs of African Men who have Sex with Men: Barriers to Healthcare and Promoting HIV and STI Treatment in Sub-Saharan Africa.”. East African Journal of Health and Science 3 (1), $59-77$. https://doi.org/10.37284/eajhs.3.1.339.

\section{HARVARD CITATION}

Ross, M. W., Kashiha, J., Misedah, L., Mgopa, L. R., Larsson, M., Agardh, A., and Venkitachalam, K. K. (2021) “Addressing the Healthcare Needs of African Men who have Sex with Men: Barriers to Healthcare and Promoting HIV and STI Treatment in Sub-Saharan Africa.”, East African Journal of Health and Science, 3(1), pp. 59-77. doi: 10.37284/eajhs.3.1.339.

\section{IEEE CITATION}

M. W., Ross, J., Kashiha, L., Misedah, L. R., Mgopa, M., Larsson, A., Agardh, \& K. K., Venkitachalam. "Addressing the Healthcare Needs of African Men who have Sex with Men: Barriers to Healthcare and Promoting HIV and STI Treatment in Sub-Saharan Africa.”, EAJHS, vol. 3, no. 1, pp. 59-77, June. 2021.

\section{MLA CITATION}

Ross, Michael W., John Kashiha, Lourence Misedah, Lucy R. Mgopa, Markus Larsson, Anette Agardh \& Krishna Kumar Venkitachalam. "Addressing the Healthcare Needs of African Men who have Sex with Men: Barriers to Healthcare and Promoting HIV and STI Treatment in Sub-Saharan Africa.". East African Journal of Health and Science, Vol. 3, no. 1, June 2021, pp. 59-77, doi:10.37284/eajhs.3.1.339.

\section{INTRODUCTION}

Sub-Saharan Africa (SSA) is a region that is characterized by a high prevalence of barriers to healthcare for men who have sex with men (MSM). MSM face high levels of stigmatization, including physical, verbal, sexual, moral abuse and related religious condemnation, from the general public as well as healthcare workers (HCWs). Homosexual behaviour is criminalized in most nations in SSA, and MSM and non-governmental organizations (NGOs) supporting them contend with governmental discrimination in policy, both at official and unofficial levels. These factors are compounded by a lack of appropriate health education and access to treatment of sexually transmitted infections (STI) and human immunodeficiency virus (HIV). The barriers to healthcare for the general population in SSA include issues related to cost, distance, transport, distribution of HCWs, HCW training, and healthcare resources. Over and above these, MSM face additional barriers that are direct or indirect results of stigma and discrimination associated with homosexuality and its criminalization. Stigmamediated direct barriers occur when patients identified as or suspected to be MSM are denied care or provided care less than the prevailing standard of care. In some cases, care is accompanied by morality-based insults, threats of reporting to law enforcement, those of religious condemnation, or public humiliation at healthcare facilities. At an indirect level, stigmatization may result in depression and internalized homophobia (IH; also referred to as internal homonegativity) among MSM as well as gaps in formal education of HCWs, which are indirect barriers to healthcare. 
The healthcare policies focusing on MSM in SSA are diverse, with some providing opportunities for healthcare delivery and others presenting obstacles. In 1996, South Africa became the first country in the world to prohibit constitutional discrimination based on sexual orientation, and MSM-specific healthcare services are currently available in urban areas (Harrisberg, 2020). Even in South Africa, there is a strong urban-rural divide in the availability of discrimination-free healthcare. Elsewhere, healthcare services publicly catering to MSM are largely non-existent. Facilities that offer services to MSM may be forced to close down, with their staff at the risk of getting assaulted or arrested. The major barrier to care is stigma and discrimination at multiple levels, from governmental policies to religious and cultural prejudices. Moreover, negative societal attitudes and beliefs against MSM may persist among HCWs thanks to the lack of education and awareness on issues of sexual orientation and gender.

Despite the homophobic environment, many healthcare professionals in SSA provide appropriate care, particularly in private practice, and remain eager to learn about sexuality-related aspects in various healthcare disciplines such as medicine, nursing, midwifery, and pharmacy. Thus, adequate education of HCWs in sexuality and related aspects is an important avenue for reducing barriers to healthcare among not only MSM but also other sexual minorities and the general population with sexual issues. We believe that the current situation in SSA can be equated to the HIV epidemic situation in the early 1980s. Because HIV had first emerged in MSM, misinformation, stigma, and discrimination against MSM were rampant. Over the past 30 years, enormous strides in dealing with sexuality have been made worldwide, and the same can be expected in SSA in the coming decades.

This review discusses the direct effects and indirect effects of stigma and discrimination on MSM in SSA, i.e., the problems related to healthcare access and treatment (including HIV testing) and the psychological outcomes such as depression and internalized homophobia, respectively. It also discusses the direct impact of criminalization on stigmatization and discrimination and notes social and geographical barriers and facilitators specific to MSM in SSA. Finally, the SPEND model is proposed as a framework for improving healthcare for MSM in SSA, along with the discussion of examples of effective educational interventions for HCWs in SSA. Although parallel barriers exist for lesbians, this review considers them beyond its scope because of the additional and sometimes unique factors involved.

\section{Terminology}

Discussions on sexual minorities in SSA must factor in the terminologies and labels used. Terms such as "gay" and "bisexual" have different meanings in SSA, and models analysing MSM behaviour in SSA through the lens of the Western model of gay culture and sexual diversity are of limited explanatory value. Moen et al. (2014), in a study on the terminology used for MSM in Tanzania, report that "gay" is used to refer to receptive partners or those whose appearances and mannerisms typical of female gender roles, whereas "guy" is used for penetrative partners or those with appearances and mannerism typical of male gender roles. This is typical of SSA, where gender role-linked labelling and classification is the norm. MSM in SSA are also more likely to be bisexual and/or married or cohabiting with a woman. Hence, "men who have sex with men" is a more suitable label to describe this subpopulation in SSA, notwithstanding the significance of sexual identity labels such as homosexual, bisexual, or heterosexual gender identities (Young \& Meyer, 2005).

\section{DIRECT EFFECTS OF STIGMA AND DISCRIMINATION}

In this section, we review the studies that have reported on the direct effects of the homonegative environment in SSA on the provision and access to healthcare for STIs by MSM. We provide a special focus on HIV testing and treatment because sexual transmission is the most common form of HIV transmission in SSA.

\section{Limitations to Healthcare Access and Treatment}

Criminalization and stigmatization of homosexuality are important barriers to providing access and utilization of health services by professionals, indirectly contributing to poorer 
health status among MSM, as noted in a systematic literature review by Albuquerque et al. (2016) regarding access to health services for Lesbian Gay Bisexual Transgender (LGBT) individuals. In SSA, more factors may be in play to complicate this situation. One example is from Tanzania, where homosexuality is illegal and punishable. In 2016, the Tanzanian Government threatened legal repercussions to NGOs providing HIV care support to MSM, and offered support only to those entities that are in line with the country's law, traditions, and customs (Mwalimu, 2016). This may exclude healthcare services to MSM and other key populations, including heterosexual populations, contributing to the HIV epidemic in SSA (Mpondo, Gunda \& Kilonzo, 2017).

\section{Healthcare Denial and Ill-Treatment by HCWs}

The problems related to healthcare access and treatment for MSM in Tanzania are relatively well documented. Magesa et al. (2014) interviewed 50 MSM aged 18-24 recruited by snowball sampling from a faith-based organization in Dar es Salaam, Tanzania. In this sample, $88 \%$ of MSM were single with no children, $56 \%$ were bisexual, and $38 \%$ were unemployed. Emerging themes identified from the study included factors related to healthcare access denial, of which stigma and discrimination were paramount. Most participants were afraid to seek healthcare for HIV and STIs; if they sought help, they were not free to reveal the MSM-associated symptoms, such as rectal or even oral symptoms. Participants gave examples of healthcare staff rebuking patients in front of their colleagues or revealing their sexual orientation to other patients. Participants reported HIV counsellors storming out of sessions, abusive language use by HCWs, refusal of services because of their sexual orientation, and being lectured on the evils of homosexuality. Some were told that they will go to hell and that their illness is a curse for having homosexual sex. Participants reported decreasing the frequency of HIV testing or traveling long distances to go to more accepting clinics because of such behaviour. These data are consistent with the findings from South Africa and other SSA countries reported by Müller (2016) and Lane et al. (2008), respectively, who also report that such breaches of privacy and confidentiality are barriers to HIV testing. These findings are confirmed by Ogunbajo et al. (2018) in
Ghana. They report that barriers to MSM accessing treatment included fear of being seen, poor finances, and health system challenges. Motivators included having social support, and fear of death from HIV/AIDS, while facilitators were having health insurance, familiarity with hospital personnel, and having had positive experience in a clinic.

Stigma theory is one of the best frameworks for conceptualizing barriers to healthcare in MSM in SSA. Larsson et al. (2016b) tellingly titled their paper on the healthcare experiences of MSM in Dar es Salaam, Tanzania "Being forced to become your own doctor," highlighting the fact that MSM in SSA have to conduct their own medical diagnosis and treatment because of the related stigma. The findings of their study, based on the qualitative content analysis of 12 in-depth interviews of respondents randomly selected from Ross et al.'s 2013 respondent-driven sampling (RDS) study, parallel those of Magesa et al. (2014), which used a similar methodology. Larsson et al. reported MSM facing significant stigmatization for treatment associated with HIV or STIs after revealing their sexual orientation, including outright rejection from healthcare facilities. Other themes revealed in the study included the recurring experience of stigmatization of MSM in healthcare, which was characterized as "(MSM) becoming third-class citizens in healthcare." MSM were treated as objects of humiliation, with accounts of HCWs calling in other staff to stare at or comment on them, along with the use of "evil" and "unnatural" to describe them (Larsson et al., 2016b). HCWs also walked out and refused to treat MSM, sometimes even threatening to have them killed. MSM reported increased levels of distrust toward HCWs and internalization of the resultant shame.

Concerns regarding confidentiality and privacy in HIV-testing facilities were also reported in Magesa et al.'s (2014) Tanzanian study. Participants gave examples of healthcare staff rebuking patients in front of their colleagues or revealing their sexual orientation to other patients. They also reported reducing HIV-testing frequency or traveling long distances to visit friendlier clinics. These results were consistent with the findings from South Africa and other SSA countries reported by Müller (2016) and Lane et al. (2008), respectively, who also report 
that such breaches of privacy and confidentiality are barriers to HIV testing.

\section{Threat to Healthcare Facilities Catering to MSM}

Larsson et al. (2016a; 2016b; 2017) also note that political, social, and religious factors in Tanzania combine to pose a threat for healthcare facilities catering to MSM. Given the laws, religious beliefs, and culture of Tanzania, clinics dedicated to MSM testing and treatment could be threatened or attacked. Similarly, healthcare professionals offering clinical evaluation and treatment for MSM report the repercussions they may face considering the criminalization of homosexuality in Tanzania. However, this argument is not persuasive because treatment is rarely refused for patients involved in other criminalized activities, such as fighting, driving under the influence, or abortion.

\section{Misinformation and Falsification of STI Symptoms}

A considerable proportion of the STI disease burden among MSM in SSA remains unreported. Because of the stigma, MSM delay revealing or conceal STI symptoms, and rarely divulge anal symptoms. Penile symptoms are more readily revealed, despite the associated shame, as they do not indicate homosexual activity.

In their Tanzanian study, Larsson et al. (2016a) discuss the theme of MSM misinforming HCWs in government-run facilities regarding STI symptoms to avoid being mistreated; for example, reporting penile symptoms even if they suffered from rectal problems. Given that HCWs tend to administer treatment based on symptoms without a detailed clinical examination or ancillary laboratory tests, MSM may not receive any treatment because of the lack of penile discharge. If HCWs insisted on further examination or testing, MSM may find excuses to misguide HCWs and abscond so that anal symptoms remain concealed. Although MSM stated that this problem is largely absent in private clinics, they tended to seek public hospitals and dispensaries for treatment despite the potential mistreatment because of expenses.

In an RDS study involving 200 MSM in Dar es Salaam, Ross et al. (2016) evaluated the correlations between specific STI-related symptomatology and HCW behaviour. MSM having conditions homosexuality-associated condition with "poor deniability" (e.g., anal sores, anal discharge, oral sores) stated that they were more likely to over-thecounter treatment or not seek any treatment. Respondents also reported a high prevalence of rude HCW behaviour for STI symptoms, with the highest prevalence for anal discharge, swelling of the groin, and oral sores. This indicates that, compared to overall STIs, treatment for homosexuality-related STIs is even more inadequate.

Interestingly, Niang et al.'s (2003) study in Dakar, Senegal reported anal bleeding and discharge in $22 \%$ of participants, with $42 \%$ also reporting penile discharge. The prevalence of penile and anal sores was similar at $24 \%$ and $22 \%$, respectively. MSM tended to ignore anal symptoms or administer selftreatment with over-the-counter medications or prescription-based ones shared by friends.

These studies make it clear that falsification of medical histories and delayed treatment are factors promoting STI and HIV transmission, and the mitigation of stigma and discrimination through education are important steps in controlling STI and HIV transmission in MSM populations.

\section{Limited Reporting of MSM Behaviour}

MSM identity or behaviour is under-reported in SSA because of stigma and discrimination. A snowball sampling survey of $252 \mathrm{MSM}$ in Lesotho by Baral et al. (2011) found that only $24.4 \%$ had disclosed their homosexual behaviour to HCWs. Moreover, $25.2 \%$ reported being married at some point in their life. HCWs, because of inadequate sexual education, may preclude MSM behaviour in MSM with positive marital history. Larsson et al. (2016a; 2016b; 2017) in multiple studies among the Tanzanian MSM population also report that the inability to divulge STI symptoms, especially anal ones, because of the fear of revealing sexual orientation was a clear barrier to appropriate diagnosis and treatment.

\section{Visibility Conundrum}

Larsson et al.'s (2016b) research also brought up the "seesaw of visibility" theme. MSM reported being forced to balance their need to remain discreet and 
the need to seek proper and timely treatment, especially for STI/HIV. In SSA, this remains an issue even in non-healthcare contexts among MSM who are noticeable by their attire or mannerisms, forcing even the less-effeminate ones to dress inconspicuously. Ross et al. (2020) found that HCW discrimination against MSM was based largely on whether they were perceived as having inappropriate gender roles, than their behaviour of having sex with other men.

\section{Violence and Social Rejection}

Studies from West Africa have long confirmed that stigma in the region is no different than in other parts of Africa, providing ubiquitous MSM-related challenges not limited to healthcare. Niang et al.'s (2003) study was among the first studies on MSM and HIV in SSA that highlighted the discriminatory environment that MSM face. This study included a convenience sample of 250 MSM aged over 18 years, and provides evidence of factors affecting MSM beyond healthcare characterized by stigma, violence, and rejection. Forty-three percent of participants reported being raped, with 13\% reporting being raped by police. Respondents also reported reluctance in seeking treatment for STI symptoms and being abused or denied treatment when they sought help.

\section{Alternative Sources for Healthcare}

The existing evidence regarding MSM in SSA should serve as a major warning sign that key populations are being driven away from public healthcare and are being forced to create their own alternatives because of stigma and discrimination. Agardh et al. (2017), in their qualitative interview study involving $15 \mathrm{MSM}$ in Dar es Salaam who used pharmacies/drugstores regularly for sexual health needs, made an important point that alternatives will informally evolve (or will need to be developed) in the health sector if formal medical services are perceived as untrustworthy and unprofessional. Hence, a detailed discussion of the various factors in play is warranted.

Agardh et al. (2017) reported a theme of MSM needing to balance the risk of exposing themselves against treatment needs even while approaching the pharmacists. This mirrors the dilemma MSM face while seeking treatment at public clinics because of HCW mistreatment. With pharmacists, however, MSM reported feeling safer to reveal their symptoms, with a lower tipping point of treatment needs outweighing the associated risks.

Ross et al. (2015) reported that social and sexual networks play a key role in MSM seeking healthcare from alternative sources through pharmacies/drugstores. Agardh et al. (2017) also discussed the theme of strategies MSM employed to ensure safety while seeking alternative sources of healthcare. MSM sought information from social networks and observed the drugstore owners or pharmacists as they interact with other customers to gauge their possible reaction and the potential of non-discriminatory service. Apart from entrusting pharmacists to remain discreet, MSM had the option to visit pharmacies/drugstores at idle times for ensuring privacy. In addition, pharmacies/drugstores were also perceived to be more accessible than hospitals due to a combination of being universally available, geographically proximal, and friendly.

Larsson et al. (2016a) brought up a major theme of MSM in Tanzania considering government-run clinics as a last resort or a barrier to early or timely treatment for STI symptoms. This contributes to MSM seeking over-the-counter treatment from pharmacies (with licensed pharmacists)/drugstores (selling common medications, including antibiotics) or needing to resort to self-treatment. For the former, MSM preferred to either approach pharmacy/drugstore staff who were known to be professional or understanding, or purchase medications themselves after consulting friends about appropriate treatment. A related qualitative study involving in-depth qualitative interviews of 16 pharmacists and drugstore workers in Dar es Salaam provides further insights on their perceptions and experiences providing STI treatment to MSM (Larsson et al., 2016b). Over time, pharmacists and drugstore workers reported developing an emotional connection with MSM clients, which facilitated treatment provision. However, it also undermined objective decisionmaking when MSM approached them without prescriptions. 


\section{Regulation of Alternatives}

It is clear that an environment characterized by limited possibilities for diagnosis and treatment and the perceived untrustworthiness and lack of confidentiality in public clinics has generated a migration of MSM from government-run clinics to the more "trustworthy" alternative of pharmacies/drugstores. In this scenario, inadequate prescription of treatment is a potent threat to the spread of STIs. Hence, the factors associated with it require further analysis.

Inadequate treatment may be prescribed because pharmacies/drugstores need to prioritize for-profit services or because of inadequate knowledge among pharmacists and drugstore owners about appropriate treatment regimens. Further, pharmacists are forced to prescribe treatments that MSM could afford, even if these may prove to be ineffective. Finally, measures for regulation of such prescriptions may impact current service provision function, further compromising the STI-treatment status among MSM. All of these factors should be adequately addressed for addressing the needs of MSM for STI treatment in SSA. Providing education to pharmacists and drugstore workers a potential solution to barriers for STI treatment in government-run clinics is a potential solution.

\section{Legal Barriers for MSM and Solutions}

The criminalization of homosexuality in most parts of SSA compounds the healthcare access issues for MSM, as they have limited legal recourse for remedial action. Even in South Africa, where homosexuality is decriminalized and legal recourse is available, discrimination persists. Müller (2016) reported frequent discrimination, ridicule, or denial of service by HCWs among patients from sexual and gender minorities in South Africa. This underlines the limitations of laws in changing social attitudes. However, positive themes have also emerged, with Lane et al. (2008) reporting accounts of MSM in South Africa challenging the abuse of HCWs. Some participants also noted increasing levels of tolerance toward sexual-minority groups resulting from the related legal reforms. On the other hand, legality does still not automatically ensure an accepting climate. In Rwanda, Adedimeji (2019) noted in their qualitative study that despite
MSM behaviour not being criminalized, options for sexual wellbeing in MSM were limited and mostly clandestine because of high social stigma. Nevertheless, an environment upholding legal and human rights will ultimately, over time, curb the abuse and healthcare denial to sexual minorities. Focus on HCW attitudes and beliefs as well as decriminalization is needed.

\section{Convergence of Factors}

Individual and societal factors can coexist and converge to create more complex barriers to healthcare for MSM in SSA. Wanyenze et al. (2016) conducted a qualitative study using semi-structured in-depth interviews with 85 Ugandan self-identified MSM recruited by snowball sampling to investigate barriers and opportunities for increasing HIV treatment services in Uganda. Sixty-two (72.9\%) MSM stated that they were not comfortable communicating about their sexual practices and related illnesses to providers, and $69(81.1 \%)$ felt that providers did not respect them, with 44 (51.8\%) experiencing difficulties in accessing health services. The researchers identified the following nine major barriers to healthcare for MSM: (1) unwelcome provider behaviours and discrimination; (2) limited provider skills and knowledge to handle MSM-related issues; (3) negative community perceptions toward MSM; (4) fear of exposure as MSM; (5) limitations in access to MSM-specific services; (6) high mobility of MSM population, particularly sex workers; (7) lack of high-level guidelines for providers in dealing with MSM; (8) an unfavourable legal environment; and (9) fears related to HIV-associated stigma.

Many of these barriers are modifiable, especially in the longer term. Specifically, promoting dialog among and within key populations, including individual MSM, MSM community groups, and health workers, so that MSM is viewed as people rather than as caricatures is an easy and productive approach to decrease discrimination and allay misconceptions.

Sandfort et al. (2008) in a community-based study of 1045 MSM in South Africa, reported that facilitators to HIV testing included older age, openness toward one's sexuality, and being homosexual rather than bisexual. Sandfort et al. 
(2015) also studied the HIV-testing practices among MSM from former South African townships, which are underdeveloped racially segregated public housing estates. Interviews with 81 MSM aged 2039 years indicated that although most had undergone HIV testing, there was considerable variation in the frequency of testing. Barriers to testing included being concerns about revealing their sexuality, anxiety regarding possible HIVpositive status and the related stress, and the need to confront their potentially risky behaviour. Because an HIV-positive status is necessary for access to the HIV treatment cascade, lack of HIV testing an important barrier.

In summary, the following common themes were identified among MSM in SSA for avoiding stigmatization in healthcare settings. Attending private clinics, consulting non-local clinics to ensure confidentiality, not revealing the site of STI symptoms, avoiding mannerisms that may reveal their sexuality, consulting a trustworthy HCW, resorting to self-medication, finding accepting pharmacies/drugstores and seeking treatment, or using social networks for advice on what drugs to buy from pharmacies/drugstores.

\section{INDIRECT EFFECT OF STIGMA AND DISCRIMINATION}

Psychological health is often seen as separate from physical health in the discussion of barriers to treatment and healthcare. However, stigma and discrimination are closely associated with clinical depression and suicidal ideation, especially among MSM. Depression, in turn, is associated with HIVand STI-risk behaviours, making it a barrier to treatment and sexual risk reduction. Numerous studies have examined these associations. In the following section, we highlight the data on depression and suicidal ideation in MSM to emphasize the links between stigma and discrimination and mental health, life-threatening conditions, risk behaviours for STIs and HIV, and seeking treatment. This is followed by a deeper analysis of the themes/factors that are in play.

\section{Depression and Suicidal Ideation}

Using RDS sampling, Risher et al. (2013) studied stigma and discrimination among 323 MSM seeking healthcare in Swaziland. A majority $(61.2 \%)$ of participants reported fear of seeking healthcare because of being MSM. Only a quarter (25.6\%) of the participants indicated that they had ever disclosed their sexual practices to HCWs. A high prevalence of depressive symptoms (58\%) and suicidal ideation (37\%) was noted among participants. Multivariate analysis showed that MSM having experienced legal discrimination as a result of sexual orientation or behaviour was significantly associated with fear of seeking healthcare. Disclosure of sexual behaviour to an HCW was associated with having completed secondary education, having used a condom with the last male partner, and suicidal ideation. Mgopa et al. (2017) found a prevalence of $70 \%$ of MSM were depressed in Tanzania, and Ulanja et al. (2019) in an RDS survey of 1301 MSM in Côte d'Ivoire, using the same instrument reported $48 \%$, with one in five severely depressed. In the same study, $22.6 \%$ of MSM said they were afraid to seek health care, and a further $15.8 \%$ avoided health care. These data indicate that a clear association between stigma and discrimination and the prevalence of depression and suicidal ideation.

\section{Unsafe Sexual Practices}

The relationship between depression and STIs among MSM in SSA also needs further analysis. Adam et al. (2005) note that MSM who are depressed are less likely to worry about unsafe behaviour. Given the association of depression with major stigma and violence, avoidance of treatment would be consistent with higher levels of depression or fatalism. The association between depression and HIV risk in MSM has been studied extensively. Ahaneku et al. (2016) reported the results of 205 MSM in Tanzania recruited by RDS; the participants were recruited from Ross et al. (2013)'s sample of 300 patients. The researchers used the Swahili version of Brief Patient Health Questionnaire mood scale (PHQ-9; range of scores 0-27), which is validated for use in East African populations. Using a - cut-off score of 4 , the prevalence of depression in the sample was $46 \%$. The study included HIV zero surveillance, and depression was associated with HIV-seropositive status, with 64 participants being HIV-seropositive. However, only 5 of the $64(7.8 \%)$ MSM were aware of their HIV-seropositive status at recruitment. 
Thus, depression was associated with high-risk behaviours for HIV, such as multiple sexual partners, but not with the knowledge of HIV seropositivity. These results indicate a link between stigma and becoming HIV infected and a lack of association between the knowledge of HIV infection and depression onset.

\section{Violence}

Mgopa et al. (2017) recruited 345 MSM in Tanzania by RDS. Using PHQ-9 as a depression measure and the same cut-off score of 4, they found that depressive symptoms were present in 70\% of MSM. Overall violence was reported by $94.2 \%$ of participants, with $90.1 \%, 73.6 \%$, and $72.5 \%$ reporting emotional, physical, and sexual violence, respectively. Participants who experienced violence had $>11$ times higher rate of depressive symptoms. Sexual partners were implicated in $66 \%$ of participants reporting sexual violence, whereas police officers were implicated in $56 \%$ of participants reporting physical violence. Community members (91\%), neighbours (88\%), and relatives (83\%) were mostly implicated in participants reporting emotional violence.

\section{Religion}

In SSA, punitive narratives espoused by many conservative religions are an integral part of the landscape of stigma and provide justifications for discrimination against MSM, which may contribute to clinical depression. This, in turn, may cause a reluctance to seek treatment and fear of further stigmatization. Ross and Anderson (2014) found that IH was moderately and anti-gay violence levels were strongly associated with depression in MSM in Tanzania. In their sample composed equally of Muslim- and Christian-identifying men, strong religious beliefs were associated with depression. The authors attribute that the strong association between religious beliefs and depression to the rationalization of the punishment-associated narrative for victims of anti-gay violence. Further research on the interaction of religion and stigma among MSM in SSA is needed.

\section{Internalized Homophobia/Homonegativity}

Internalized homophobia or homonegativity (IH) is defined as "the gay person's direction of negative social attitudes toward the self, leading to a devaluation of the self and resultant internal conflicts and poor self-regard" (Meyer \& Dean, 1998, p. 160). It is associated with depression and unsafe sexual behaviour among MSM. In Nigeria, Adebajo et al. (2012) linked IH to risk factors for HIV among MSM. In a large RDS-based sample of over 1,000 men in Lagos and Ibadan, they used a locally-constructed IH scale to analyse associations. Findings indicated that high $\mathrm{IH}$ was positively associated with being bisexual or being married to a woman, and being HIV-seropositive. Older age and being employed were also associated with high IH. The authors postulate that IH creates a vicious cycle of anxiety, substance abuse, and related other psychological factors in MSM, leading to considerable resistance to the adoption of HIVpreventive measures.

Ross et al. (2013) conducted a study including 216 Ugandan MSM recruited by RDS using an IH scale that was validated for cross-cultural use (Ross et al., 2010; Tran et al., 2018). They found that IH was significantly associated with HIV-risk behaviours. Men with higher IH were more likely to have sex under the influence of alcohol or other drugs and to have unprotected anal sex. These findings suggest a strong link between stigmatizing and homonegative environment and its impact on self-devaluation and risk behaviour. In their subsequent work in neighbouring Tanzania, Ross et al. (2014) found a $30 \%$ HIV-seropositivity rate in 300 MSM recruited using RDS. As expected, IH and depression were found as significant predictors of HIV seropositivity. This is consistent with the Ugandan data.

It is clear that healthcare-related adversities that MSM face in an environment of stigmatization and discrimination, along with the negative psychological impact such an environment creates, will lead to a wide variety of obstacles in the prevention, testing, and treatment of STIs. IH plays a critical role in this, with it likely being a crucial mediator between stigma and poor psychological adjustment and depression. 


\section{DECRIMINALIZATION OF HOMOSEXUALITY}

Most studies on MSM in SSA have been conducted in countries where homosexual behaviour is demonized and/or criminalized. Although South Africa occupies a unique place in this context - with a constitution that not only decriminalizes homosexual behaviour but also outlaws discrimination based on sexual orientationdiscrimination remains pervasive in many areas of South Africa. The following discussion highlights the impact of criminalization, why the South African experience should not be a deterrent for decriminalization in other nations in SSA, and why decriminalization is a necessary trigger to the slow process of improving healthcare access to MSM.

\section{Spread of Misinformation in the Absence of Formal Education}

A study among HCWs in Malawi conducted by Wirtz et al. (2014) found many negative attitudes and beliefs regarding MSM, including the belief that MSM could not contract HIV and STIs. The researchers also interviewed five $\mathrm{HCW}$ s regarding how MSM are treated in healthcare settings. HCWs stated the existence of an initial disbelief among them that MSM existed in Malawi and that they could contract HIV. They admitted to early resistance to even have conversations about MSM.

\section{Risk to Clinics and HCWs}

Some HCWs in Malawi interviewed by Wirtz et al. (2014) stated that healthcare providers were reluctant to administer treatment because of the lack of proper legislation for protecting MSM. HCWs believed that decriminalization-related legislative changes would make the provision of services to MSM much easier. They also reported the sensationalist media, who attacked clinics catering to MSM and the HCWs who worked at these clinics, as barriers to healthcare. HCWs were aware that denying services and treatment would lead to the spread of infection, starting a vicious cycle that cannot be easily broken. It is easy to criticize providers for attitudes toward MSM without understanding the associated complexities. Policies and laws criminalizing MSM behaviour and sensationalizing stories in the press and media not only affect MSM, but they also make it difficult for HCWs to provide clinical services without fear of backlash or even losing jobs. Hagopian et al. (2017), in an analysis of the impact of legislation and stigma on MSM in 21 African nations, note that criminalization reinforces negative views, undermines healthcare and research, and fuels the STI epidemic. They also argue that decriminalization should be a goal in the public health agenda.

\section{Abusive and Inappropriate Practice}

Criminalization creates an environment where abusive and medically inappropriate practice thrives. Cichowitz, Rubenstein and Beyrer (2018) document forced anal examinations carried out on MSM by physicians at the request of lawenforcement officials as proof of homosexuality. They report that this practice exists in at least nine countries, many of them being in SSA, with Kenya and Tunisia recently ending the practice. The researchers argue that forced anal examinations are scientifically unfounded, ethically inappropriate, and meet the United Nations' definition of torture. This report also unfortunately demonstrates the unethical and medically and scientifically inappropriate behaviour toward MSM by HCWs. The criminalization of homosexuality forces HCWs to become moral and legal guardians, often in breach of human rights and medical ethics, which in turn creates mistrust of HCWs among MSM.

\section{SOCIOCULTURAL AND GEOGRAPHICAL FACTORS AS BARRIERS AND FACILITATORS}

Awareness regarding geographical and cultural differences in SSA as well as the dynamics within MSM social networks in SSA is crucial for developing safe sites for HIV and STI diagnosis and treatment for MSM in SSA. Unlike in the West, MSM-focused treatment centres located in areas with large MSM populations may be difficult in SSA. Moreover, isolated geographical locations and smaller MSM community networks act as barriers to STI-related healthcare. Apart from the physical barriers such as distance to travel and transportation cost, a lack of "accepting" clinics in the neighbourhood is a factor. Further, in areas with smaller MSM communities, social networks are 
even more crucial for communicating health information, including information healthcare facilities where treatment could be safely sought. The following section discusses these geographical and social networking characteristics in the context of healthcare access for MSM. Several unique sociocultural factors act as barriers and facilitators in the healthcare framework for MSM in SSA. Because of the cultural and ethnic diversity of Africa, they may operate in different ways in different locations thanks to the variations in cultural, legal, and religious, administrative, and political factors. Hence, instead of classifying factors into barriers and facilitators, we note their interdependence. In other words, we consider that the absence of a barrier as a facilitator and vice versa.

\section{Sexuality and Morality}

In SSA, the stigma associated with sexuality is rooted in the widespread correlation between sexual activity and moral deficiency. The stigma that MSM experience is part of a larger moral condemnation of sexuality, which is shared with other key populations at elevated risk of HIV transmission, which is primarily through sexual contact in SSA. In MSM, the moral, religious, and cultural attitudes and beliefs about homosexuality complicate the matter further. Thus, there is a clear overlap between the stigma and discrimination-related healthcare for HIV and STIs. However, it is important to note that HIV-associated stigma is uniform among all key populations throughout SSA. Carrasco, Arias and Figueroa (2017) carried out a thematic analysis of five qualitative studies conducted in high-HIV-prevalence provinces in Mozambique between 2009 and 2012. HIV stigma was found to be a barrier to HIV testing and counselling, disclosure, partner notification, and antiretroviral therapy (ART) access and adherence in all communities, including MSM. Thus, the MSM-related stigma and discrimination appears to be a subset of a larger problem of discrimination associated with sexuality.

\section{Private versus Public Facilities}

Some participants in studies from SSA report that HCWs with neutral or positive attitudes toward MSM are more common in private healthcare facilities than in government-run ones. Although both types of facilities charge fees, the private ones charge more and offer a higher quality of care. Magesa et al. (2014) report that accessibility to HIV-related health services for MSM in Dar es Salaam, Tanzania was limited because of their inability to afford private clinic treatment or longdistance bus fares to get treatment, compounded by low HIV knowledge. Participants in Larsson et al.'s (2016a; 2016b) studies also reported that HCWs in private facilities were more accepting and professional.

\section{Discrimination based on Gender-inappropriate Behaviour and Psychological Health}

Displaying gender roles typical of the opposite sex among MSM is a potent barrier given its association with increased discrimination. This is consistent with data from South Africa reported by Sandfort et al. (2016), who reported that gender nonconformity is a variable affecting the psychological health of MSM. Perhaps gender-nonconforming individuals were less able to "pass" as heterosexuals. However, this finding also raises the question as to whether the stigma is directed toward perceived genderinappropriate behaviour, homosexual behaviour, or a combination of both. Regardless of the severity of rectal symptoms, anal examination is unlikely unless the HCW suspects homosexual behaviour.

Several reports detail the discrimination against MSM from other countries in SSA. Using snowball sampling, Fay et al. (2011) surveyed MSM in Malawi $(n=202)$, Namibia $(n=218)$, and Botswana $(n=117)$ on stigma and healthcare access. Among the overall sample, $21 \%$ of MSM reported ever being blackmailed because of their sexuality. Surprisingly, only 5\% overall MSM reported that they had been denied healthcare because of their perceived or actual sexuality. This is likely because it is difficult to know the proportion of men whose sexuality was obvious or disclosed. However, being diagnosed with STIs was associated with the history of being denied healthcare based on sexuality and the fear of reporting sexual orientation in the healthcare setting. In terms of the intersectionality of stigmatization, HIV status was the most prominent factor, with a strong relationship between receiving HIV treatment and experiencing discrimination. The effects of both direct 
discrimination (e.g., denial of healthcare and derogatory/abusive comments) and indirect discrimination (e.g., lack of sensitivity and knowledge) were noted, and there was a close relationship between fear to seek treatment and history of discrimination.

\section{The Urban-Rural Divide}

In a region as vast as SSA, it is worthwhile to note the differences among rural and urban regions regarding the stigma and discrimination that MSM face. Not surprisingly, variations exist among regions regarding these differences as well. Larsson et al. (2017) conducted a study based on qualitative interviews of MSM from Tanga, a smaller provincial Tanzanian city of about 270,000 people. The participants were randomly selected from a larger study sample recruited using RDS. The interviewed MSM stated similar challenges to those in Dar es Salaam, with societal discrimination against homosexual men spilling over to healthcare settings. MSM with financial means went to private clinics, mirroring the trend in larger cities. Participants also stated that the major concern that prevented them from seeking healthcare was the fear of exposure, in both healthcare settings and at the societal level, given the smaller, close-knit community in the city.

Other regions in SSA present different challenges. Maleke et al. (2017) conducted qualitative interviews with 23 MSM from rural South Africa using snowball sampling. The participants also reported high levels of prejudice and a homonegative environment in rural areas. The participants stated that being identified in a rural homophobic environment was a prominent concern, with participants noting that it was easier to be gay in larger towns, where anonymity and acceptance of sexual minorities are higher. Only two participants were aware that there were MSM-specific health services available in South Africa, and seeking care from a private physician was the preferred option among participants. Most participants visited doctors in neighbouring towns to protect their anonymity or would avoid seeking treatment at the local clinic fearing discrimination. Some participants also reported rude behaviour from HCWs as a deterrent to seeking treatment. The researchers note that empathy toward and understanding of MSM's actual experiences, knowledge of their social dynamics, and capacity building for cultural competence, especially in rural health services, are essential steps to mitigating HCWs' prejudices and discrimination against MSM.

This shows that the potential impact of stigma and discrimination remains similar or higher in smaller cities or rural areas. As noted before, in such a rural setting, socially vulnerable MSMs would hesitate to file complaints against unprofessional HCWs and would not even expect that their complaints may be taken seriously. HCW education regarding MSM and STIs will play a critical role in improving this situation.

\section{Geographical Spread}

A few studies have been conducted on the distribution of meeting places for MSM in SSA. Ross et al. (2012) analysed the locations of approximately 98 known meeting places for MSM in Dar es Salaam in Tanzania. These places were usually small and evenly distributed across the city, rather than in one or two "red light" areas as is typically found in the West. This geographical spread, likely related to the need to stay unnoticed by authorities or vigilante groups, makes the outreach for healthcare more difficult and makes reliance on public transport unfeasible for HCWs. This is another reason, apart from the political and safety reasons, why specialized clinical services focused on the MSM population may not be feasible in SSA.

\section{Information Sharing in Networks}

The stigma and stereotyping that prevails in SSA make information sharing especially that regarding discrimination within MSM networks a crucial factor affecting healthcare access. Participants in Larsson et al.'s (2016a, b) qualitative research in Tanzania reported wide circulation of rumours regarding healthcare-related ill-treatment among MSM in Dar es Salaam, including death threats issued to MSM by HCW at clinics. Although these narratives may be exaggerations and are unverifiable, such threats remain "real" for the MSM. They fuel further rumours among MSM regarding the reputations of facilities and HCWs, 
providing encouragement for MSM to administer self-treatment if "safer" private clinics are inaccessible. It is important to note that rumours regarding MSM thrive among $\mathrm{HCW}$ and the general public as well. Hence, education and communication of accurate information among all key populations are crucial to improving the situation.

\section{Network-based Approaches}

In contrast, a social network study of MSM in Tanzania (Ross et al., 2016) using RDS showed well-connected, complex networks across RDS trees, for both a large metropolis (Dar es Salaam) and a smaller provincial city (Tanga). This suggests that network-based treatment approaches, at least for MSM who self-identify as gay or bisexual, could be an avenue for word-of-mouth and snowball recruitment into healthcare, even in smaller cities.

\section{Use of Internet}

The use of the Internet as a medium for finding sexual partners is on the rise among MSM in SSA. A study on MSM in Tanzania indicated high levels of access to and use of electronic media and its use for healthcare information (Ross et al., 2018a). Hence, sharing information online may be a potential solution to breach the healthcare information and access barriers. Considering that information on HIV- and STI-treatment locations are shared within MSM communities, networkbased information about appropriate treatment sites could be a useful approach.

\section{THE SPEND MODEL}

As discussed above, various factors present major to challenges healthcare service provision to MSM in the homophobic environment prevailing in SSA. Nevertheless, their complex interplay offers room for the development of coherent models for improving healthcare delivery to MSM. One such model is the SPEND model developed by Ross et al. (2015) to guide the provision of healthcare services for MSM and training for HCWs.

SPEND is an acronym that refers to the important aspects of the model.
- S refers to "safe treatment sites," including that provided by NGOs and outreach, where MSM are not abused, stigmatized, denied services, or exposed to public ridicule.

- $\mathbf{P}$ refers to using "pharmacies/drugstores as treatment sources or sites," considering that MSM tend to seek treatment there when public healthcare services present the threat of abuse and private services are unaffordable.

- E stands for "education for health professionals," both formal and continuing professional training, which aims to provide information to allow them to serve key populations, including MSM, and to understand their professional and ethical responsibilities.

- $\mathbf{N}$ refers to "navigation," which refers to the provision of a peer or professional-patient "navigator" to accompany MSM to clinics. These "navigators" act as social supports or role models and steer patients through complex processes/protocols in treatment facilities, ensuring that they are not ignored by HCWs.

- D stands for "discrimination reduction" through long-term education on human sexuality and human rights and the removal of discriminatory statutes and other barriers.

\section{Aims of the SPEND Model}

The SPEND model was developed on the basis of data not only on the healthcare situation for MSM in SSA but also on the wider context of healthcarerelated discrimination worldwide. It offers an empirical framework within which barriers can be reduced and progress can be measured. By directly addressing the barriers identified at the grassroots level, the SPEND model's aims are targeted to ensure provision of medically and ethically appropriate healthcare. The model is centred around training programs for $\mathrm{HCWs}$ for overcoming barriers to healthcare of key populations in homophobic environments worldwide. The shortand long-term goals of these programs are to decrease specific barriers at all levels in the healthcare system. These goals are not limited to SSA and are designed to ensure appropriate professional conduct by HCWs toward MSM and adequate provision of healthcare to MSM. 


\section{Education for HCWs}

The discussion on mistreatment of MSM in SSA in the passages above presents a bleak picture of the prospect of change in attitudes and beliefs of HCWs. However, there is evidence to suggest that the situation is by no means monolithic and that there are significant numbers of HCWs who are prepared to set examples and act as role models, even in the face of government and social disapproval and policy barriers.

\section{Examples from Kenya}

The work of Van der Elst et al. (2013) in Kenya is a classic example of the effect of appropriate professional training relating to the medical needs and treatment of MSM. The researchers developed and evaluated the effect of web-based sensitivity training on $74 \mathrm{HCWs}$ in Kenya with the aim of reducing homonegative prejudice. They assessed the feasibility of such training interventions to improve HCWs' knowledge and attitudes. HCWs showed significant and sustainable increases in knowledge and decreases in homophobia score compared to the baseline at 3 months of follow-up. Increases in knowledge were associated with a reduction of negative attitudes toward MSM, and the positive impact of the intervention was strongest in the HCWs who had the lowest knowledge scores and/or more extreme negative attitudes toward MSM at baseline. The positive changes were also strongest in HCWs in clinical roles in government settings. Among the most positive outcomes was the willingness of HCWs, many of whom had negative attitudes and beliefs about MSM at baseline, to learn about sexual health, and the intervention succeeded in improving their knowledge and changing their attitudes. The researchers conclude that, while improving knowledge is the first step, practical experience in treating MSM will further improve HCW attitudes and beliefs. Further, these trained HCWs will act as informal educators and role models of other HCWs in the same clinical settings.

\section{Examples from Tanzania}

The participants in Larsson et al.'s (2016a; 2016b; 2017) studies in Tanzania report a steep learning curve for HCWs regarding MSM-associated factors. This indicates that HCW education regarding LGBT-related aspects could alleviate many of the problematic attitudes exhibited by HCWs toward MSM. Ross et al. (2018b) conducted a Tanzanian study using a related approach with a more comprehensive curriculum covering the broad field of sexual health. The study involved a two-day sexual health workshops, focusing on sexual health generally but also including information on MSM, using a curriculum developed by a large US university in conjunction with a UN agency. The study's results made it clear that HCWs in SSA are eager to learn about sexual health and how to take sexual histories. Matched pre- and post-workshop evaluations showed extensive significant improvements in knowledge, and participants' attitudes changed significantly on questions relating to same-sex and transgender issues. The enthusiastic reception of these workshops both at both school and student levels indicates that a substantial proportion of healthcare professionals in SSA are aware of their lack of knowledge in sexual health, including the aspects related to key populations, and are eager to learn and make amends.

\section{CONCLUSIONS}

The expression "justice delayed is justice denied" is common in legal circles. In healthcare, treatment delayed in some conditions is not only treatment denied but may also be a death sentence. In SSA, barriers to healthcare are a matter of health and justice. Where the barriers are focused on denial of healthcare, they directly facilitate the spread of disease within key populations. This may rise to the level of doing harm, unintentional or intentional, in contravention of the Hippocratic oath. It is clear that there is a human rights component to some barriers to healthcare for MSM. In the early days of the HIV epidemic in North America, the slogan "Silence = Death" was widely used. In SSA, a variation of this, i.e., "Stigma $=$ Death," rings true.

Where discrimination exists in a population, discriminative attitudes will likely permeate HCWs at similar levels to that in the rest of the community. However, HCWs are held to a higher standard considering that they (and the universities, colleges and professional bodies that train them) rise above the discrimination and are expected to be educated and professional. To remedy the situation, professional bodies and educational institutions 
must include sexual health in their curricula. Reported evidence strongly suggests that HCWs are eager to receive such education. Although there are no quick fixes to problems of stigma and discrimination, appropriate professional training of HCWs, in all areas of health including sexual health, should be considered an essential part of health and human rights aid by external donors and by professional bodies in SSA. It is promising to note that leading African universities have taken up this challenge and have piloted such courses (Ross et al., 2018).

To summarize this review, a large body of research exists regarding the widespread stigma and discrimination across SSA in healthcare settings, including denial of services and treatment, lack of confidentiality, and abuse of patients by HCWs. This has forced MSMs to provide false clinical and personal histories, avoid treatment altogether, seek out treatment alternatives such as advice from peers of seeking treatment at pharmacies/drugstores, or seek treatment at private clinics when they can afford it. These trends are fuelled by a toxic environment of stigmatization and criminalization of MSM behaviours, media sensationalism regarding MSM, discriminatory health policies, religion-based attacks, and human rights abuses. Research studies involving HCWs confirm the lack of knowledge and skills resulting from the lack of adequate training. The resultant mistreatment drives MSM them toward alternative treatment sources or avoid treatment altogether, continuing the spread of STIs. In this situation, the positive results from pilot interventions for increasing the knowledge and reducing homophobic attitudes of HCWs offer considerable encouragement. It is proof that appropriate training of HCWs can address many of these negative drivers of stigma and health service discrimination. However, such interventions must also be extended to other less direct, but important, sources of barriers, such as religious leaders, politicians, and journalists, who inform key opinion leaders.

The situation of MSM in SSA can be largely equated to the experiences of gay and bisexual populations affected by the HIV epidemic in the 1980s. Even back then, training and role modelling in health settings had strong and positive impacts on clinical responses, service provision, and policies toward the key populations, resulting in the improvement of skills to deal with and change in attitudes toward them. The promising effect of interventions on HCW education in SSA strengthens the parallels between the response to the HIV epidemic in its early stages and the situation in SSA. This should hopefully strengthen the focus on well-established interventions and modelling of appropriate behaviour to educate and reform HCWs, which will improve the healthcare settings for MSM in SSA. We also must not repeat the mistakes made or forget the lessons we learned in the 1980s in the conduct of early HIV prevention, screening, and treatment campaigns. Although the data on stigma and discrimination against MSM in SSA are consistently overwhelming, the data on the possibilities of effective and productive knowledge and skills training HCWs in SSA are equally promising. Hence, the focus of efforts should remain on reducing stigma and discrimination against MSM in healthcare settings by implementing training interventions for HCWs and extending such interventions to tackle other barriers.

\section{ACKNOWLEDGMENT}

This work was supported by the Swedish Research Council under Grant 2014-2649

\section{REFERENCES}

Adam, B. D., Husbands, W., Murray, J., \& Maxwell, J. (2005). AIDS optimism, condom fatigue, or self-esteem? Explaining unsafe sex among gay and bisexual men. Journal of sex research, 42(3), 238- 248. doi: 10.1080/002244 90509552278.

Adebajo, S. B., Eluwa, G. I., Ahonsi, B. A., Allman, D., \& Myers, T. (2012). Prevalence of internalized homophobia and HIV associated risks among men who have sex with men in Nigeria. African Journal of Reproductive Health, 16(4), 21-28. https://www.ajol.info/in dex.php/ajrh/article/view/83669

Adedimeji, A., Sinayobye, J. D., Asiimwe-Kateera, B., Chaudhry, J., Buzinge, L., Gitembagara, A., Murenzi, G., Mugenzi, P., Patel, V. V., Castle, P. E., Mutesa, L., Palefsky, J., \& Anastos, K. M. 
(2019). Social contexts as mediator of risk behaviors in Rwandan men who have sex with men (MSM): Implications for HIV and STI transmission. PLoS ONE, 14(1), Article e0211099. https://doi.org/10.1371/journal.pone. 0211099

Agardh, C., Weije, F., Agardh, A., Nyoni, J., Ross, M.W., Kashiha, J., and Larsson, M. (2017). Using pharmacists and drugstore workers as sexual healthcare givers: A qualitative study of men who have sex with men in Dar es Salaam, Tanzania. Global Health Action, 10, 1389181, doi: 10.1080/16549716.2017.1389181

Ahaneku, H., Ross, M. W., Nyoni, J. E., Selwyn, B., Troisi, C., Mbwambo, J., Adeboye, A, and McCurdy, S. (2016). Depression and HIV risk among men who have sex with men in Tanzania. AIDS Care, 28(suppl. 1), 140-147. doi: 10.1080/09540121.2016.1146207

Albuquerque, G. A., de Lima Garcia, C., da Silva Quirino, G., Alves, M. J. H., Belém, J. M., dos Santos Figueiredo, F. W., ... \& Adami, F. (2016). Access to health services by lesbian, gay, bisexual, and transgender persons: systematic literature review. BMC International Health and Human Rights, 16(1), 1-10. doi: 10.1186/s12914-015-0072-9.

Anderson, A. M., Ross, M. W., Nyoni, J. E., \& McCurdy, S. A. (2015). High prevalence of stigma-related abuse among a sample of men who have sex with men in Tanzania: implications for HIV prevention. AIDS Care, $27(1)$, 63-70. doi:10.1080/09540121.2014.951597

Baral, S., Adams, D., Lebona, J., Kaibe, B., Letsie, P., Tshehlo, R.,... \& Beyrer, C. (2011). A crosssectional assessment of population demographics, HIV risks and human rights contexts among men who have sex with men in Lesotho. Journal of the International AIDS Society, 14(1), 36. doi:10.1186/1758-2652-1436

Carrasco, M. A., Arias, R., \& Figueroa, M. E. (2017). The multidimensional nature of HIV stigma: evidence from Mozambique. African
Journal of AIDS Research, 16(1), 11-18. doi:10.2989/16085906.2016.1264983

Cichowitz, C., Rubenstein, L., \& Beyrer, C. (2018). Forced anal examinations to ascertain sexual orientation and sexual behavior: An abusive and medically unsound practice. PLoS medicine, 15(3), e1002536. Doi: 10.1371/journal.pmed.1002536.

Fay, H., Baral, S. D., Trapence, G., Motimedi, F., Umar, E., Iipinge, S.,... \& Beyrer, C. (2011). Stigma, health care access, and HIV knowledge among men who have sex with men in Malawi, Namibia, and Botswana. AIDS and Behaviour, 15(6), 1088-1097. doi:10.1007/s10461-0109861-2

Hagopian, A., Rao, D., Katz, A., Sanford, S., \& Barnhart, S. (2017). Anti-homosexual legislation and HIV-related stigma in African nations: what has been the role of PEPFAR?. Global health action, $10(1), \quad 1-10 . \quad$ Doi: $10.1080 / 16549716.2017 .1306391$

Harrisberg, K. (2020, March 10). South Africa opens continent's first queer wellness centre. Reuters. https://www.reuters.com/article/ussafrica-lgbt-health-trfn/south-africa-openscontinents-first-queer-wellness-centreidUSKBN20X32A

Lane, T., Mogale, T., Struthers, H., McIntyre, J., \& Kegeles, S. M. (2008). "They see you as a different thing": the experiences of men who have sex with men with HCWs in South African township communities. Sexually Transmitted Infections, $84(6)$, 430-433. doi: 10.1136/sti.2008.031567

Larsson, M., Pettersson, K. O., Kashiha, J., Ross, M. W., \& Agardh, A. (2016a). Stretching the boundaries: Tanzanian pharmacy workers' views and experiences of providing STI services for men who have sex with men. PloS one, 11(11), e0166019. doi: 10.1371/journal.pone.0166019.

Larsson, M., Ross, M. W., Månsson, S. A., Nyoni, J., Shio, J., \& Agardh, A. (2016b). Being forced to become your own doctor: men who have sex with men's experiences of stigma in the

74 | This work is licensed under a Creative Commons Attribution 4.0 International License. 
Tanzanian healthcare system. International Journal of Sexual Health, 28(2), 163-175. doi: 10.1080/19317611.2016.1158763

Larsson, M., Shio, J. M., Ross, M. W., \& Agardh, A. (2017). Acting within an increasingly confined space: a qualitative study of sexual behaviours and healthcare needs among men who have sex with men in a provincial Tanzanian city. PloS One, 12(8), e0183265. doi: 10.1371/journal.pone.0183265

Magesa, D. J., Mtui, L. J., Abdul, M., Kayange, A., Chiduo, R., Leshabari, M. T., ... \& Tungaraza, D. (2014). Barriers to men who have sex with men attending HIV related health services in Dar es Salaam, Tanzania. Tanzania Journal of Health Research, 16(2), 1-10. doi: 10.4314/thrb.v16i2.8

Maleke, K., Makhakhe, N., Peters, R. P., Jobson, G., De Swardt, G., Daniels, J.,... \& Struthers, H. (2017). HIV risk and prevention among men who have sex with men in rural South Africa. African Journal of AIDS Research, 16(1), 31-38. doi:10.2989/16085906.2017.1292925

Meyer, I., \& Dean, L. (1998). Internalized homophobia, intimacy and sexual behaviour among gay and bisexual men. In: Herek, G (Ed.), Stigma and Sexual Orientation: Understanding Prejudice against Lesbians, Gay Men, and Bisexuals. Thousand Oaks: Sage.

Mgopa, L. R., Mbwambo, J., Likindikoki, S., \& Pallangyo, P. (2017). Violence and depression among men who have sex with men in Tanzania. BMC psychiatry, 17(1), 1-5. Doi: 10.1186/s12888-017-1456-2

Moen, K., Aggleton, P., Leshabari, M. T., \& Middelthon, A.-L. (2014). Gays, guys, and mchicha mwiba: same-sex relations and subjectivities in Dar es Salaam. Journal of Homosexuality, 61(4), 511-539. doi:10.1080/00918369.2014.865457

Mpondo, B. C., Gunda, D. W., \& Kilonzo, S. B. (2017). HIV epidemic in Tanzania: the possible role of the key populations. AIDS Research and Treatment. doi: 10.1155/2017/7089150
Müller, A. (2016). Health for all? Sexual orientation, gender identity, and the implementation of the right to access to health care in South Africa. Health and human rights, 18(2),

195-208 https://www.ncbi.nlm.nih.gov/pmc/articles/PM C5395001/pdf/hhr-18-195.pdf

Mwalimu, U. (2016, Oct 27). Statement by the Minster for Health, Community Development, Gender, Elderly and Children, HON. Ummy Mwalimu on key populations HIV services in Tanzania. Ministry of Health, Community Development, Gender, Elderly, and Children, The United Republic of Tanzania. https://m.facebook.com/afyatz/posts/123092286 3594845 .

Niang, C. I., Tapsoba, P., Weiss, E., Diagne, M., Niang, Y., Moreau, A. M., \& Castle, C. (2003). 'It's raining stones': stigma, violence and HIV vulnerability among men who have sex with men in Dakar, Senegal. Culture, Health \& Sexuality, $5(6)$, 499-512. doi:10.1080/1369105031000152715

Ogunbajo, A., Kershaw, T., Kushwaha, Boakye, F., Wallace-Atiapah, N.D., \& Nelson, L. (2018). Barriers, Motivators, and Facilitators to Engagement in HIV Care Among HIV-Infected Ghanaian Men Who have Sex with Men (MSM). AIDS Behav 22, 829-839 (2018). https://doi.org/10.1007/s10461-017-1806-6

Risher, K., Adams, D., Sithole, B., Ketende, S., Kennedy, C., Mnisi, Z.,... \& Baral, S. D. (2013). Sexual stigma and discrimination as barriers to seeking appropriate healthcare among men who have sex with men in Swaziland. Journal of the International AIDS Society, 16(3S2), 18715. doi:10.7448/IAS.16.3.18715

Ross, M. W., Kajubi, P., Mandel, J. S., McFarland, W., \& Raymond, H. F. (2013). Internalized homonegativity/homophobia is associated with HIV-risk behaviours among Ugandan gay and bisexual men. International journal of STD \& AIDS, 24(5), 409-413. doi: $10.1177 / 0956462412472793$.

Ross, M. W., Kashiha, J., Nyoni, J., Larsson, M., \& Agardh, A. (2018a). Electronic media access and

75 | This work is licensed under a Creative Commons Attribution 4.0 International License. 
use for sexuality and sexual health education among men who have sex with men in four cities in Tanzania. International Journal of Sexual Health, 30(3), 264-270. Doi: 10.1080/19317611.2018.1491919

Ross, M. W., Larsson, M., Jacobson, J., Nyoni, J., \& Agardh, A. (2016). Social networks of men who have sex with men and their implications for HIV/STI interventions: Results from a crosssectional study using respondent-driven sampling in a large and a small city in Tanzania. BMJ Open, 6(11), e012072. doi:10.1136/bmjopen-2016-012072

Ross, M. W., Larsson, M., Nyoni, J. E., \& Agardh, A. (2017). Prevalence of STI symptoms and high levels of stigma in STI healthcare among men who have sex with men in Dar es Salaam, Tanzania: a respondent-driven sampling study. International Journal of STD \& AIDS, 28(9), 925-928. doi:10.1177/0956462416683625

Ross, M. W., Nyoni, J., Bowen, A. M., Williams, M. L., \& Kashiha, J. J. (2012). Sexual and geographic organisation of men who have sex with men in a large East African city: opportunities for outreach. BMJ open, 2(6), e001813. doi:10.1136/bmjopen-2012-001813

Ross, M. W., Nyoni, J., Larsson, M., Mbwambo, J., Agardh, A., Kashiha, J., \& McCurdy, S. A. (2015). Health care in a homophobic climate: the SPEND model for providing sexual health services to men who have sex with men where their health and human rights are compromised. Global health action, 8(1), 26096. Doi: 10.3402/gha.v8.26096.

Ross, M.W., \& Anderson, A. M. (2014). Relationships between importance of religious belief, response to anti-gay violence, and mental health in men who have sex with men in East Africa. In Piedmont, R. L. \& Village, A. (eds), Research in the Social Scientific Study of Religion, (Vol 25, pp. 160-172). Leiden: Brill.

Ross, M. W., Kashiha, J., \& Mgopa, L.R. (2020). Stigmatization of men who have sex with men in health care settings in East Africa is based more on perceived gender roleinappropriate mannerisms than having sex with men. Global health action, 13(1), DOI: 10.1080 /16549716.2020.1816526

Ross, M. W., Leshabari, S., Rosser, B. R. S., Trent, M., Mgopa, L., Wadley, J., ... \& Agardh, A. (2018b). Evaluation of an assessment instrument for a sexual health curriculum for nurses and midwifery students in Tanzania: The sexual health education for professionals scale (SHEPS). Applied Nursing Research, 40, 152156. doi: 10.1016/j.apnr.2018.01.005

Ross, M.W., Smolenski, D.J., Kajubi, P., Mandel, J.S., McFarland, W., and Raymond, H.F. (2010). Measurement of internalized homonegativity in gay and bisexual men in Uganda: Cross-cultural properties of the Internalized Homonegativity scale. Psychology, Health and Medicine, 15, 159-165.

Sandfort, T. G., Nel, J., Rich, E., Reddy, V., \& Yi, H. (2008). HIV testing and self-reported HIV status in South African men who have sex with men: results from a community-based survey. Sexually transmitted infections, 84(6), 425-429.

Sandfort, T., Bos, H., Knox, J., and Reddy, V. (2016). Gender nonconformity, discrimination, and mental health among black South African men who have Sex with Men: A further exploration of unexpected findings. Archives of Sexual Behaviour, 45, 661-670. Doi 10.1007/s10508-015-0565-6

Sandfort, T., Knox, J., Collier, K.L., Lane, T., \& Reddy, V. (2015). HIV testing practices of South African township men who have sex with men in the era of expanded access to ART. AIDS and Behaviour 19, 561-574. Doi 10.1007/s10461014-0843-7

Tran, H., Ross, M. W., Diamond, P. M., Berg, R. C., Weatherburn, P., \& Schmidt, A. J. (2018). Structural validation and multiple group assessment of the Short Internalized Homonegativity Scale in homosexual and bisexual men in 38 European countries: Results from the European MSM Internet Survey. Journal of Sex Research, 55(4-5), 617-629. doi 10.1080/00224499.2017.1380158 
Ulanja, M.B., Lyons, C., Ketende, S., Diouf, D., Kouamé, A., Ezauarchi, R., Bamba, A., Drame, F., Liestman, B., \& Baral, S. (2019). The relationship between depression and sexual health service utilization among men who have sex with men (MSM) in Côte d'Ivoire, West Africa. BMC Int Health Hum Rights 19. Doi:10.1186/s12914-019-0186-6

Van der Elst, E. M., Smith, A. D., Gichuru, E., Wahome, E., Musyoki, H., Muraguri, N., ... \& Sanders, E. J. (2013). Men who have sex with men sensitivity training reduces homoprejudice and increases knowledge among Kenyan healthcare providers in coastal Kenya. Journal of the International AIDS Society, 16, 18748. doi: 10.7448/IAS.16.4.18748

Wanyenze, R. K., Musinguzi, G., Matovu, J. K., Kiguli, J., Nuwaha, F., Mujisha, G.,.. \& Wagner, G. J. (2016). "If you tell people that you had sex with a fellow man, it is hard to be helped and treated": barriers and opportunities for increasing access to HIV services among men who have sex with men in Uganda. PloS One, 11(1), e0147714. doi: 10.1371/journal.pone.0147714

Wirtz, A. L., Kamba, D., Jumbe, V., Trapence, G., Gubin, R., Umar, E.,... \& Baral, S. D. (2014). A qualitative assessment of health seeking practices among and provision practices for men who have sex with men in Malawi. BMC International Health and Human Rights, 14(1), 20. doi:10.1186/1472-698X-14-20

Young, R. M., \& Meyer, I. H. (2005). The trouble with "MSM" and "WSW": Erasure of the sexual-minority person in public health discourse. American journal of public health, 95(7), 1144-1149. doi: 10.2105/AJPH.2004.046714 